\title{
Multi Drug Therapy- Combination of Structural Inhibitors to Eradicate Leprosy
}

\author{
Vandana Kashyap ${ }^{1}$, Astha Pandey ${ }^{2}$, Swati Tripathi ${ }^{3}$ \\ ${ }^{1}$ Department of Biotechnology, Institute of Engineering and Technology, Bundelkhand University, Jhansi, UP- 284128, India
}

\begin{abstract}
Being persistant infection, caused by Mycobacterium leprae, Leprosy is affecting about 50,000 annually. It affects Schwann cells (the glial cells of peripheral nervous system) and macrophages. Its long incubation period (5-7 years) classify leprosy as paucibacillary $(\mathrm{PB})$ and multibacillary $(\mathrm{MB})$ depending on the bacillary load. Recent advances have been made in developing molecular diagnostics, in identifying and designing highly effective treatment. MULTI DRUG THERAPY (MDT) has revamped leprosy with the combination of rifampicin, clofazimine and dapsone (MB patients) and rifampicin and dapsone (PB patients). Various new agents like fluroquinolones (moxifloxacin), rifapentine, macrolides and monocycline in varying combination have also proved their potency. Drugs like thalidomide analogues, pentoxifyline, selectively inhibit cytokine and control type-2 reactions (erythema nodosum leprosum reaction) which have association with deposition of tissue and circulation of immune complex. Present study discusses about the high potential effect of MDT over other treatments through complex structural comparison and their interactions.
\end{abstract}

Keywords: Mycobacterium leprae, Schwann cells, Paucibacillary, Multibacillary, erythema nodosum leprosum

\section{Introduction}

Leprosy, granulomatous chronic infection leads to neurologic disabilities and physical deformities. Recent researches has shown that Mycobacterium leprae, the causative agent induces lipid droplet formation in infected macrophages, cholesterol accumulates in ML-infected macrophages that can be referred as potential target for drugs $^{[1]}$. Demyelination of PNS is induced upon attachment of M. laprae to schwann cells where bacteria hides until the infection fully establishes and then spread causing excess chemokines release $^{[1]}$. WHO-MDT with high effectiveness combines Dapsone, rifampin and clofazimine targets at folate biosynthesis pathway, $\beta$-subunit of RNA polymerase and DNA respectively, thus inhibiting replication and transcription in M.leprae ${ }^{[3]}$.

\section{Methods and Materials}

Different Drug's structures (Dapsone, Rifampicin, Clofazimine and Monocycline) are developed in RasMol through online smiles translator, the mode of action of these drugs ( disscussed below) on M. Leprae that causes leprosy were studied from reserach articles and WHO report to make a comparitive study of drugs which are used today. This knowledge could be helpful for the researchers to use them for increasing the effectiveness of treatments or developing new drugs and early diagnosis.

\section{Result and Discussion}

Paucibacillary(PB) and Multibacillary(MB) are two different types of leprosy- number of $M$. leprae in Multibacillary is high whereas in Paucibacillary is small, skin smear test is negative in $\mathrm{PB}$, while it is positive in MB. Monotheraphy of DAPSONE was used to treat patients. But resistant-strain against dapsone became a problem and thus MDT treatment emerged which controlled the spread of drug-resistant strains $^{[3]}$. Dapsone, 4,4-diaminodiphenyl sulphonesynthetic sulphone, structurally and functionally related to suphonamide drugs. It targets folate biosynthesis pathway of
M. leprae by acting as competitive inhibitor of $p$ aminobenzoic acid (PABA $)^{[3]}$. Rifampicin,3-[[(4-methyl-1piperazinyl)-imino]-methyl a bactericidal component. Target $\beta$-subunit of the RNA polymerase encoded by $\operatorname{rpoB}^{[2]}$. Clofazimine, [3-p-chloroanilino)-10(p-chlorophenyl)-2, 10dihydro-2-(isopropyl imino) phenazine] - substituted iminophenazine, have anti-mycobacterial and antiinflammatory activity. Highly lipophilic binds preffentially to mycobacterial DNA at base sequences containing guanine $^{[3]}$. Monocycline, (7-dimethylamino-6-demethyl-6deoxytetracycline) a bactericidal and its mechanism is thought to be similar to all tetracycline which act by inhibiting protein synthesis. It binds reversibly to $30 \mathrm{~S}$ ribosomal subunit and blocks the binding of amino acyl RNA transferase to the messenger RNA ribosomal complex ${ }^{[1]}$.

In this review we have discussed about potential drugs used in Multi Drug Therapy, the most effective treatment for leprosy which have various targets proving leprosy as a curable disease today.
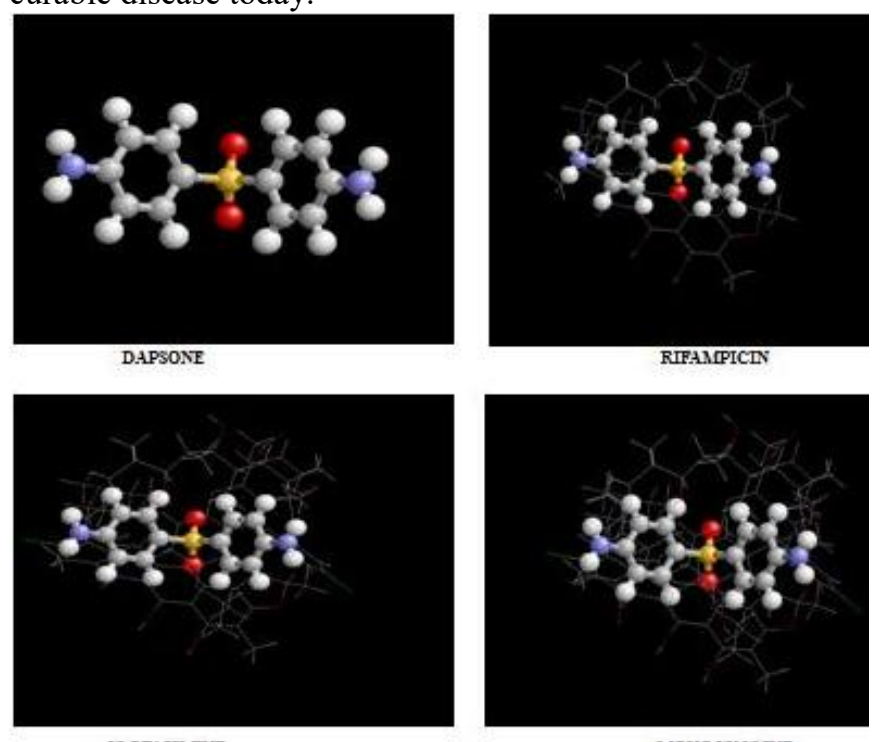

CLOFAZTMINT

MONOCYCLINP

Figure 1: Structure of MDT drugs

\section{Volume 4 Issue 12, December 2015}




\section{International Journal of Science and Research (IJSR) \\ ISSN (Online): 2319-7064}

Index Copernicus Value (2013): 6.14 | Impact Factor (2014): 5.611

\section{Conclusions}

Leprosy, most dreaded disease is no longer incurable through Multi drug therapy due to its all-round treatment by using combination of effective drugs.

\section{Acknowledgements}

I forward my gratitude to Dr. Anand Kumar Pandey (Assistant Professor) Department of Biotechnology, Institute of Engineering and Technology, Bundelkhand University, Jhansi. It would have been impossible for us to do the needful without his kind support and guidance.

\section{References}

[1] Moschela SL. (2004) An update on the diagnosis and treatment of leprosy, 51, 417-426.

[2] Rees RJ, Pearson JM, Waters MF. (1970) Experimental and clinical studies on rifampicin in treatment of leprosy, 88, 89-92.

[3] Boerrigter G, Ponnighaus JM, Fine PEM.(1998)

[4] Preliminary appraisal of WHO recommended multiple drug regimen in paucibacillary leprosy patients in Malawi, 56, 408. 